\title{
ÁNFORAS ALTO, MEDIO Y BAJOIMPERIALES PRODUCIDAS EN EL ALFAR DE PUENTE MELCHOR (VILLANUEVA, PASO A NIVEL: PUERTO REAL, CÁDIZ)
}

\author{
ROMAN AMPHORAE FROM THE PUENTE MELCHOR KILN \\ (VILLANUEVA, PASO A NIVEL: PUERTO REAL, CÁDIZ)
}

\author{
por \\ ENRIQUE GARCÍA VARGAS \\ $M^{a}$ LUISA LAVADO FLORIDO
}

\begin{abstract}
RESUMEN Presentamos en estas páginas el estudio preliminar del material anfórico producido en el alfar de Puente Melchor, también conocido como Villanueva, paso a nivel (Puerto Real, Cádiz), un establecimiento que se mantuvo activo durante casi cuatrocientos años, desde la época de Augusto hasta la de Constantino.
\end{abstract}

\begin{abstract}
A preliminary repport of the roman amphorae made in the potter's workshop Puente Melchor (also known as Villanueva, paso a nivel) in Puerto Real (Prov. Cádiz) is presented in this paper. The workshop remained making pottery four centuries, from the age of Augustus to that of Constantinus.
\end{abstract}

\section{INTRODUCCIÓN}

Durante los meses de Mayo y Junio de 1994 se procedió, a instancias de la Delegación de Cultura de Cádiz, a la excavación arqueológica de urgencia en el sector de la finca denominada Puente Melchor afectado por la inminente construcción de la variante de la Carretera Nacional IV entre El Puerto de Santa María y Puerto Real. El yacimiento se encuentra situado en el Km. 667 de la Nacional IV, sólo dos Kms. al SE de Puerto Real, extendiéndose a ambos lados de la calzada, a la altura de paso elevado que le da nombre y que salva el cruce con la línea del ferrocarril Madrid-Cádiz. Las excavaciones de 1994 se limitaron a la zona situada en la margen derecha de la carretera, en dirección a Puerto Real; pero en la margen contraria, junto a la antigua venta Nuestra Señora de Fátima, 
pueden verse al menos dos montículos correspondientes a hornos cerámicos junto a un tercero que ha servido de vertedero de una cercana fábrica de ladrillos, aún en funcionamiento ${ }^{1}$.

El topónimo Puente Melchor es reciente en la bibliografía sobre hornos anfóricos (Lazarich et alii 1991: $98, \mathrm{n}^{\circ} 11$ ), aunque corresponde a un yacimiento conocido desde 1946 con el nombre de Paso a nivel (Pemán 1959: 169 y fig. 1), por haberse hallado restos anfóricos en la zona con motivo de la construcción del puente que sustituyera al antiguo paso a nivel. $\mathrm{M}^{\mathrm{a}} \mathrm{J}$. Jiménez Cisneros designó este alfar de manera diferente en cada ocasión: “... antiguo paso a nivel del ferrocarril” (1971: 149), “... puente... en la carretera, antes de llegar a Puerto Real (1971: 150), Paso a nivel (1971: lám. LXIII), Huerta paso a nivel (lám. LXIV). Años más tarde, M. Beltrán denominó la zona como Villanueva, paso a nivel del ferrocarril (1977: 111), mientras que D. P. S. Peacock la reseña con su nombre original de Paso a nivel (Peacock 1974: 329).

\section{EL ALFAR}

La intervención de 1994 ha sacado a la luz estructuras aún en estudio correspondientes a dos hornos cerámicos rodeados por un muro de ladrillos, un tercer horno más pequeño que amortiza los anteriores, un gran vertedero de ánforas delimitado por muretes de mampostería y fragmentos de ánforas, instalaciones industriales con piletas de decantación de arcillas, conducciones de agua y habitación de tornos, así como los restos de un cuarto horno de reducidas dimensiones y función desconocida.

\section{Los hornos:}

Los de mayores proporciones se hallan rodeados de muros de ladrillos y fragmentos de ánforas. Ambos son circulares y construidos en ladrillo. El situado más al Norte conserva el laboratorio, la parrilla sobre bóveda de ladrillos sustentada en un pilar central, la cámara de cocción y el praefurnium. Junto a él, existe otro, también circular, que presenta la particularidad de llevar la parrilla sustentada sobre un pasillo central, continuación del praefurnium, formado por dos muros paralelos que sostienen una bóveda apuntada. Ambos hornos muestran huellas de haber sido reparados en varias ocasiones lo que supone un largo periodo de actividad.

Un tercer horno de menores dimensiones amortiza las estructuras que rodean los anteriores. Sólo ha sido excavado el pasillo de acceso al laboratorio, que ha librado algunas ánforas del tipo Keay XXIII (Almagro 51C).

\section{El vertedero:}

Al Norte de los hornos, más allá del muro perimetral de éstos, se ha excavado un vertedero de ánforas delimitado por muros poco consistentes y trazado sinuoso, que presenta varias fases de vertidos, prolongadas a lo largo de doscientos años.

1. Este sector ha sido objeto de una nueva Intervención Arqueológica de Urgencia en los primeros meses de 1996, dirigida, como la de 1994 por $\mathrm{M}^{\mathrm{a}}$ Luisa Lavado Florido. 


\section{Las instalaciones del alfar:}

Junto a la carretera, al Sur de los hornos se excavó una habitación estrecha con una pileta de signinum en el interior, amortizada en una segunda fase. La estructura, por su poca anchura, puede ser identificada con una habitación de torneado de ánforas (Peacock 1982: passim).

En el exterior, cerca de los hornos, se hallaron dos piletas más excavadas en el terreno natural que aún se encontraban llenas de arcilla decantada. Se documentaron también conducciones de signinum relacionadas con el abastecimiento de agua al alfar.

La estratigrafía del yacimiento se encuentra aún en fase de estudio por su excavadora. Nos limitaremos aquí a dejar constancia de las fases de actividad del alfar:

Fase I: Sólo detectada en un sondeo estratigráfico. No ha sido excavada en extensión.

Fase II: A la que corresponden los niveles de base de los vertederos, los dos hornos mayores y la construcción de las instalaciones alfareras.

Fase III: Sólo documentada en el vertedero, con restos cerámicos asociados a un muro de contención de vertidos.

Fase IV: Los niveles superficiales del vertedero corresponden a esta fase de la que se documentan también estructuras del alfar.

Fase V: Correspondiente a la fase de uso de los hornos pequeños y al último momento de ocupación de las instalaciones industriales. La habitación de torneado es remodelada y la pileta de signinum inutilizada por un muro. Igualmente se detecta la remoción de las conducciones de agua.

\section{LAS ÁNFORAS ${ }^{2}$}

Constituyen la casi totalidad de las producciones del alfar y se fabricaron en todas las fases de actividad de éste. Para la fase IV se detecta además la producción de cerámica común.

La fase I de Puente Melchor, la más antigua conocida, ha ofrecido escasos restos de ánforas del tipo Dressel 7 de tipología antigua, similares a los conocidos en otros establecimientos alfareros de la bahía de Cádiz. Junto a ellos, destaca la presencia de fragmentos atípicos de terra sigillata itálica. Todo ello nos sitúa en época augústea, más concretamente en torno al cambio de Era.

La fase II ha podido excavarse en una extensión mayor y ofrece un panorama morfológico muy diversificado en el que se incluyen los siguientes tipos:

- Dressel 7 evolucionadas (fig. 1: 1), similares a las halladas en Vindonissa (Ettlinger y Simonet 1952: fig. 26, $\mathrm{n}^{\circ}$ 586) o a bordo de los pecios Port Vendres II (Colls et alii 1977: fig. 15, $\mathrm{n}^{\circ}$ 1) y Tour-Sainte-Marie (Tchernia 1969: 498, fig. 53 G).

- Dressel 8 (fig. 1: 2) y Dressel 9 (fig. 1: 3 y 7), muy fragmentadas, lo que no permite mayores precisiones tipológicas.

- Dressel 10 (fig. 1: 5), también fragmentarias; pero en las que la morfología del borde y la tendencia de las asas se acercan a las características de las Dressel 10/Longarina 2 (Hesnard 1980: 147-8, pl. V. 3), a veces mal llamadas Dressel 24 (Cardoso 1978: 70).

- Dressel 12 (fig. 1: 4), de amplia boca, cuello bitroncocónico y cuerpo fusiforme, próximas tipológicamente a los ejemplares de Pompeya (Manacorda 1977: tav. LVI, 17).

2. Los dibujos que ilustran este trabajo son de Luís Aguilera Rodríguez y Enrique García Vargas. El Dr. Eduardo Ferrer Albelda se tomó el trabajo de entintarlos y tramarlos. 
— Dressel 17 (figs. 1: 6 y 2: 2), de cuerpo ahusado y bordes rectos, muy similares a las del Pecio Gandolfo (Blánquez y Roldán 1989: fig. 5; Martínez Maganto, inédito).

- Pivotes (fig. 2: 5) y bordes (fig. 2: 3-4) de Dressel 20. Éstos últimos se encuadran tipológicamente entre las producciones de la segunda mitad del s. I d. C., según la clasificación de St. Martin-Kilcher para Augst (1983 fig. 3).

- Beltrán IIa de cuello cónico (fig. 3: 1).

- Beltrán IIb de tipología antigua (fig. 3: 2), con transición marcada entre borde y cuello y asas insertadas por debajo del borde (García 1996: e. p.).

- Haltern 70 (fig. 2: 1), de las que algunos fragmentos no ilustrados presentan boca acampanada, característica ésta de los ejemplares de mitad de siglo en adelante: Port Vendres II (Colls et alii 1977: fig. 13), Tour Sainte-Marie (Tchernia 1969: fig. 53 E), Augst (Martin-Kilcher 1994: fig. 162: 2). Cabe destacar que es la primera vez que se documenta con seguridad la producción del tipo en la bahía de Cádiz, lo cual se confirma a la vista de fallos de horno procedentes del alfar.

En conjunto, nos encontramos ante un grupo de ánforas de muy diversa morfología. Sin embargo, el estado de desarrollo en el que se hallan los diversos tipos, algunos de los cuales (es el caso de las Dressel 7-11) tocan a su fin, mientras otros (las Beltrán Ilb, p. e.) comienzan prácticamente ahora, permiten proponer una fecha dentro de la segunda mitad del s. I d. C., más cerca, quizás de época flavia, si atendemos a la similitud de las ánforas de esta fase con la de otros alfares béticos con la misma datación, como es el caso de los hornos de El Manganeto, en Almayate Bajo, Málaga (Arteaga 1985: 184)

La fase III de Puente Melchor se caracteriza desde el punto de vista de la tipología de las ánforas por la desaparición de los ejemplares del grupo 7-11, de las Dressel 12, que reaparecerán más tarde al igual que las Dressel 20, de las Dressel 17 y las Dressel 20. Continúa el resto de las producciones, en concreto:

- Beltrán IIa similares a las de la fase II, aunque de tipología evolucionada con las asas más curvadas

(fig. 3: 3), muy cercanas tipológicamente a las halladas a bordo del pecio Tiboulen de Maïre (Pomey et alii 1987-88: fig. 14c), así como otros ejemplares de formas robustas y cuello cilíndrico (fig. 3 : 4), del tipo de las que transportó la nave Lavezzi 1 (Liou 1990: 132-133).

- Beltrán IIb de borde biselado y asas que arrancan de la boca (fig. 3: 5), también similares a las de Tiboulen de Maïre (Pomey et alii 1987-88: fig. 14d).

Estamos, pues, ante un conjunto de ánforas que tipológicamente remite a la primera mitad del s. II d. C., datación similar a la que ofrecen algunos fragmento de TSCA de la forma 6A de Hayes.

Durante la fase IV reaparecen las Dressel 12 y 20, a la vez que se despliega una morfología novedosa que culminará en la fase siguiente en la que las ánforas de Puente Melchor serán totalmente extrañas a la tradición alfarera local.

- Las Dressel 12 se presentan ahora con formas atípicas y bordes exageradamente desarrollados, sobre todo en su mitad inferior (fig. 5: 3-4).

- Las Dressel 14 (fig. 5: 1), aparecen en esta fase con ejemplares muy próximos tipológicamente a las 14b lusitanas (Cordeiro, 1990: fig. 32, $\mathrm{n}^{\circ} 10$ ).

- Las Dressel 20 vuelven a producirse, esta vez con cuellos cortos y bordes biselados al exterior (fig. 6: 5). 
- Aparecen nuevos tipos de contenedor que provisionalmente denominamos Puerto Real 1, con dos variantes (figs 4: 1 y 5: 2), la segunda de las cuales es la forma que Martin-Kilcher (1994: fig. 170, $\mathrm{n}^{\circ}$ 10) ha denominado Augst 30) y Puerto Real 2 (fig. 4: 2). Ambos tipos parecen ser formas de transición entre las Beltrán IIb y las Keay XVI.

- Se documenta la producción de imitaciones de ánforas extrabéticas (fig. 4: 3), a medio camino entre las Galoise 4 (Laubenheimer, 1985: passim) y las Dressel 30 mauritanas (Keay 1984: 95ss).

- Se imitan igualmente ánforas africanas de los tipos Keay IV -Africana IIA- (fig. 6: 3) y V -Africana IIA con gradino- (fig. 6: 2).

- Se documentan también las Keay XVI -Almagro 50- (fig. 6: 1) y las primeras Keay XXIII -Almagro $51 \mathrm{c}-$, con borde triangular.

Los vertidos de la fase IV de Puente Melchor parecen haberse formado a lo largo de un periodo de tiempo relativamente prolongado entre las últimas décadas del s. II d. C. (moneda de Marco Aurelio, TSC A de la forma 9B) y las primeras del s. III (ánforas Keay IV y V). En Augst, las ánforas de la forma Augst 30 (Puerto Real 1B) se fechan en la segunda mitad del s. II d. C. y primeros decenios del III d. C. (Martin-Kilcher, 1994: 401).

La fase $\mathrm{V}$ supone un cambio radical en la tipología de los contenedores gaditanos. Desaparecen las formas derivadas de la tradición alfarera local (Dressel 12, Puerto Real 1 y 2, Keay XVI) y los tipos producidos no serán sino imitaciones de ánforas africanas o galas o formas derivadas de ellas.

- Entre las primeras, tenemos las Keay VI (fig. 6: 6), que imitan ánforas contemporáneas de la Bizacena (Zevi y Tchernia 1969).

- Entre las segundas, contamos con dos tipos que parecen proceder de las Galoise 4 o las Dressel 30. Se trata de las Keay XXIII -Almagro 51 c- (fig. 6: 4), que comparecen ahora con algunas diferencias morfológicas con respecto a las de la fase anterior, y de las Beltrán 68 (fig. 6: 7), cuya procedencia geográfica era desconocida hasta ahora, aunque se le suponía un origen bético (Bernal 1995).

La cronología para esta fase viene dada por una moneda de Constantino I que nos sitúa entre 324 y 337 como fecha post quam.

\section{TECNOLOGÍA}

Pastas de cocción oxidada continua, duras y no muy porosas, de fractura irregular y colores rojo claro (M 37), rosa (M 69, M 70) amarillo (L 90) y verde en fallos de cocción (M 89) ${ }^{3}$, arenosas, con partículas dispersas de mineral de hierro de más de $5 \mathrm{~mm}$ y a veces ligeros engobes blanquecinos en la superficie. Al microscopio, se distingue una matriz de arcilla que incluye hasta un $20 \%$ de arena y en cuyo seno se aprecian inclusiones no muy abundantes de cuarzo subredondeado o redondeado y, en menor cantidad, subangulares de feldespato, de entre 0.03 y $0.05 \mathrm{~mm}$. También se encuentran presentes fragmentos de calcita, mica, plagioclasasas y foraminifera, así como arenisca.

Los ejemplares más recientes presentan pastas rojizas con cocción simple o doble y mayor número de inclusiones calcáreas. En efecto, en Puente Melchor se aprecian pequeñas diferencias en la composición de las pastas según las fases de producción: las últimas dos fases presentan, junto a las arcillas "tradicionales" un buen número de ejemplares con pastas de color rojo claro (M 37) que, aún conservando

3. Todas las referencias remiten al Code des colours des sols de E. Cailleaux. 
la matriz arenosa con escasos granos de cuarcita redondeados, tienen ahora inclusiones rojizas más frecuentes (hematites, cerámica reutilizada) y, sobre todo, un más alto porcentaje de calcita, presente tanto en forma de pequeñas partículas redondeadas como en filamentos paralelos o aislados (fendas).

La variedad de coloración y apariencia visual de las arcillas con que se confeccionaron las ánforas de Puente Melchor puede reconducirse, pues, a dos grandes grupos que se distinguen en la coloración (rosa, amarillo, marrón rojizo, verde, en el primero de ellos; invariablemente rojizo o marrón rojizo, en el segundo) y en la apariencia óptica (mayor cantidad de mica y partículas calizas en el segundo, que ocasionalmente presenta vacuolas amarillas redondeadas) y cuya existencia parece responder a la utilización de dos clases de barros de formación terciaria y cuaternaria, respectivamente.

No creemos que la diferenciación sea debida a factores de tipo técnico: menor temperatura de cocción para las ánforas del segundo grupo, lo que habría propiciado una transformación incompleta de la caliza hacia el grupo wollastonita-ghellenita. En efecto, los análisis de difracción de rayos $\mathrm{X}^{4}$, todos ellos efectuados sobre muestras del primer grupo, parecen descartar esta hipótesis, puesto que piezas horneadas a muy diversa temperatura, $\mathrm{y}$, por tanto, con porcentajes de calcita diferentes, tienen una apariencia muy similar.

Las ánforas de Puente Melchor presentan un acabado relativamente cuidado en las producciones más antiguas. Los contenedores de las últimas fases (IV y V) tienden a presentar una confección más defectuosa, tanto en los ejemplares de las formas Puerto Real 1 y 2 y Keay XVI, muy robustas, con superficies sin alisar y puntos de unión visibles, sobre todo en la inserción de las asas, como en los más livianos de las formas Keay I y Keay XXIII, de torneado defectuoso e incluso asas asimétricas. Por contra, los escasos fragmentos de Keay IV-VI y Beltrán 68 han sido realizados con más cuidado, presentando estas últimas un cuerpo con acanaladuras muy marcadas.

\section{EPIGRAFÍA}

Todos los sellos de Puente Melchor corresponden a la cuarta de las fases y se encontraron sobre ánforas de las formas Dressel 12 y Puerto Real 1 y 2.

- CON? (En cartela cuadrada sobre el cuello de Dressel 12)

- SOC (En Cartela cuadrada sobre Puerto Real 1 y 2 normalmente bajo el asa).

- SOCI (En cartela cuadrada sobre ánforas Puerto Real $1 \mathrm{~A}$ veces dos sellos contiguos en la misma pieza, bajo el asa y en el cuello)

- CL. SOC (En cartela cuadrada bajo el asa).

- FEX (En cartela cuadrada sobre el cuello de Puerto Real 2.

- LVC (En cartela cuadrada bajo el asa de Puerto Real 1).

- BV (En cartela cuadrada en pivote de Puerto Real 1).

- HER (En cartela rectangular, sobre forma indeterminada).

\section{VALORACIÓN}

El alfar romano de Puente Melchor ha ofrecido una estratigrafía del máximo interés que permite seguir el desarrollo formal de las ánforas gaditanas a lo largo de cuatro siglos. Situado en la misma

4. Realizadas por los Drs. Manuel y $\mathbf{M}^{\mathrm{a}}$ Carmen González, Catedrático y Profra. Titular de Química Inorgánica de la Universidad de Sevilla, a quienes agradecemos desde estas páginas su ayuda desinteresada. 
línea de costa y próximo al trazado de la Vía Augusta no parece haberse limitado a producir ánforas salsarias, sino que desde la segunda fase al menos se documentan contenedores olearios (Dressel 20) y vinarios (Hatern 70). Pero será sobre todo a partir de fines del s. II cuando se produzca un incremento en las cantidades relativas ánforas para vino (ahora Galoise IV-Dressel 30) y aceite (Dressel 20, Keay IV-V). En los últimos momentos de actividad, se siguen produciendo ánforas vinarias (Beltrán 68) junto a las dedicadas a contener las tradicionales salsas saladas de pescado (Keay XXIII y tal vez Keay VI).

Lo diverso de la producción anfórica, a la que hay que añadir cerámica común, las dimensiones del lugar, la riqueza del repertorio epigráfico, con la mención de una posible societas y la presencia en el mismo de elementos extraños al ámbito rural como una tessera de plomo empleada supuestamente como ficha de acceso a unos baños públicos (Lagóstena 1993) permiten plantear la posibilidad de que las instalaciones de Puente Melchor sean algo más que las dependencias industriales de una villa marítima. En otro lugar (García e. p.), uno de nosotros ha expuesto el proceso que en la bahía de Cádiz conduce desde una industria alfarera casi de autoconsumo, dispersa en diversos predios, hasta la concentración de las actividades cerámicas en lugares desvinculados de la producción del contenido (vici?) de los que el yacimiento de Puente Melchor creemos que es una buena ilustración.

Por otra parte, resulta del mayor interés la constatación en un alfar de la bahía de Cádiz de la producción de ánforas Dressel 20, supuesta sobre la base de la existencia en el testaccio del control fiscal Ad Portum (Chic 1985: 113), y Haltern 70, así como de formas tardías hasta ahora completamente desconocidas en la bahía de Cádiz. Destaca, finalmente, el hallazgo de instalaciones industriales asociadas a los hornos que merecen un estudio aparte.

\section{BIBLIOGRAFÍA}

ARTEAGA, O. (1985): "Los hornos romanos del Manganeto, Almayate Bajo (Málaga). Informe preliminar", Noticiario Arqueológico Hispano 23: 175-89.

BELTRÁN, M. (1970): Las ánforas romanas en España, Zaragoza.

- (1977): "Problemas de la morfología y del concepto histórico-geográfico que recubre la noción tipo. Aportaciones a la tipología de las ánforas béticas", Méthodes classiques et méthodes formelles dans l'étude des amphores. Actes du Colloque de Rome, 27-29 Mai 1974. Coll. de l'École Française de Rome 32 Roma: 97-31.

BERNAL, D. (1995): "Las ánforas del tipo Beltrán 68 en Hispania: problemática y estado de la cuestión", XXIII CNA (Elche 1994) Zaragoza.

BLÁNQUEZ J. y ROLDÁN, L. (1991): "Prospecciones arqueológicas subacuáticas en la costa de Almería", Anuario Arqueológico de Andalucía'89 III, Sevilla: 323-28.

CARDOSO, G. (1978): "Ánforas romanas no Museo do Mar (Cascais)", Conimbriga XVII: 64-91. CHIC, G. (1985): Epigrafía anfórica de la bética I. Écija.

COLLS, D; ÉTIENNE, R; LEQUÉMENT, R; LIOU, B; MAYET; F. (1977): L'epave Port Vendres II et le commerce de la Bétique a l'époque de Claude, Archaeonautica 1.

CORDEIRO, J. M. (1990): "Porto dos Cacos: uma oficina de produçao de ânforas romanas no vale do Tejo", en Mayet, F Y Alarçao, A. (eds.), As ânforas lusitanas. Tipologia. Produçao. Comércio. Coimbra: 117-51

ETTLINGER, E; SIMONET, C. (1952): Romische Keramik aus dem Stugel von Vindonissa., Basilea. GARCÍA, E. (e. p.): La produción de ánforas en la bahía de Cádiz en época romana (ss. II a. C.-IV d. C.) Memoria de licenciatura. Sevilla. 
HESNARD, A. (1980): "Un depôt augustéen d'amphores a la Longarina, Ostie", Memoirs of the American Academy in Rome XXXVI: 141-56.

JIMÉNEZ CISNEROS, M. Ja. (1971): Historia de Cádiz en la Antigüedad. Cádiz.

KEAY, S. (1984): Late Roman Amphorae in the Western Mediterranean. A typology and Economic Study: the Catalan Evidence. BAR Int. Ser. 196 (Oxford, 1984).

LAGÓSTENA, L. (1993): "Una tésera de plomo hallada en el yacimiento romano de Puente Melchor (Puerto Real, Cádiz), Habis 24: 307-9.

LAUBENHEIMER, F. (1985): La production des amphores en Gaule Narbonnaise. París.

LAZARICH, M.; ALONSO, C; LADRÓN DE GUEVARA, I.; SÁNCHEZ, M.; RODRÍGUEZ, M.: (1991): "Informe preliminar de la primera campaña del proyecto de prospección arqueológica sistemática de la campiña sur gaditana: Término de Puerto Real", Anuario Arqueológico de Andalucía'89 II, Sevilla: $98-100$.

LIOU, B. (1990): "Le commerce de la Bétique au Ier. siècle de notre ère. Notes sur l'épave Lavezzi 1 (Bonifacio, Corse du Sud)", Archaeonautica 10: 125-155.

MANACORDA, D. (1977): "Anfore spagnole à Pompei”, en L'instrumentum domesticum à Ercolano e Pompei nella prima età imperiale. Quaderni di cultura materiali 1: 121-32.

MARTIN-KILCHER, St. (1983): "Les amphores romaines à huile de la Bétique (Dressel 20 et 23) d'Augst (Colonia Aaugusta Rauricorum) et Kaiseraugst (Castrum Rauracense). Un rapport préliminaire", en Producción y Comercio del Aceite en la Antigüedad. Segundo Congreso Internacional, Madrid: 337-47.

(1994): Die Römischen Amphoren aus Augst und Kaiseaugst. Forschungen in Augst 7/2. Augst.

MARTÍNEZ, J. (inédito): El yacimiento submarino de "pecio Gandolfo". Producción y comercialización naval de salazones béticas en época altoimperial. Memoria de Licenciatura. Madrid.

PEACOCK, D. P. S. (1974): “Amphorae and the Baetican fish industries", Antiquaries Journal 54: 232-43.

- (1982): Pottery in the Roman World. An Ethnoarchaeological Approach. Londres: 232-43.

PEMÁN, C. (1959): "Alfares y embarcaderos romanos en la provincia de Cádiz", AEspA XXXII: 169-73.

POMEY, P; LONG, L.; L'HOUR, M; RICHEZ, F. (1987-88): "Recherches sous-marines", Gallia informations: 1-78.

TCHERNIA, M. A. (1969): "Direction des recherches archéologiques sous-marines”, Gallia 27: 466-99.

ZEVI, F.; TCHERNIA, M. A. (1969): “Amphores de Byzacène au Bas- Empire”, Antiquités Africaines

3: $173-214$. 

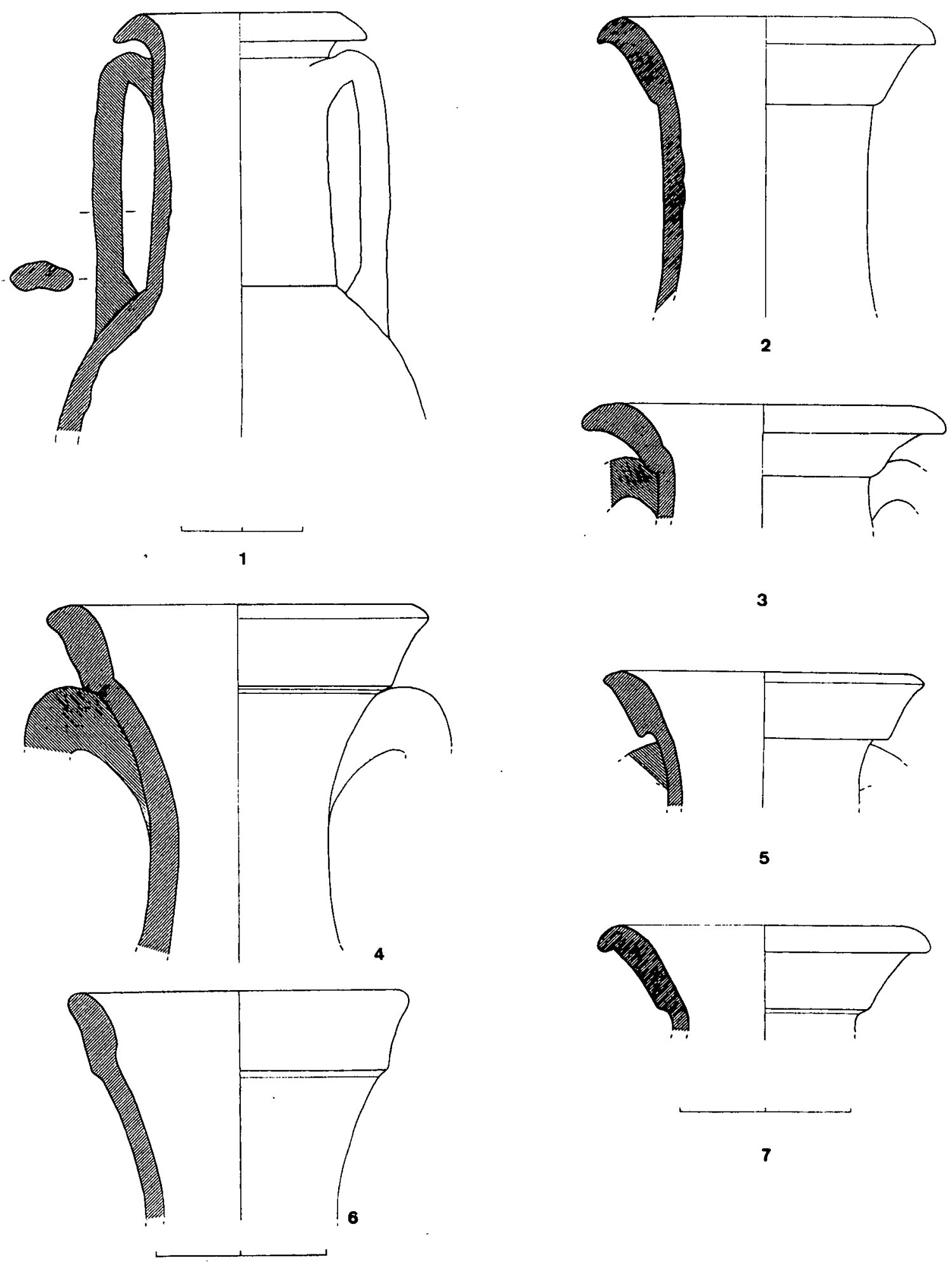

3

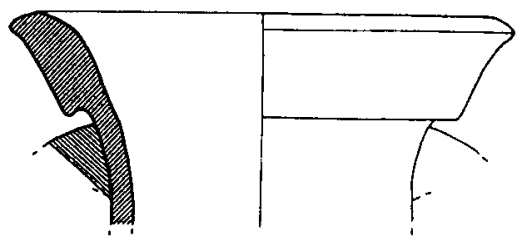

5

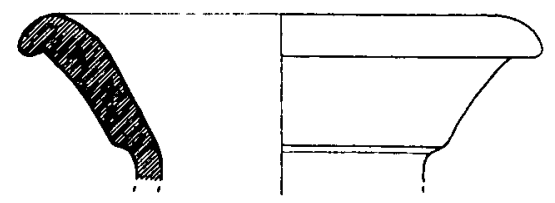

Fig. 1. Puente Melchor. Fase II: 1-7. 

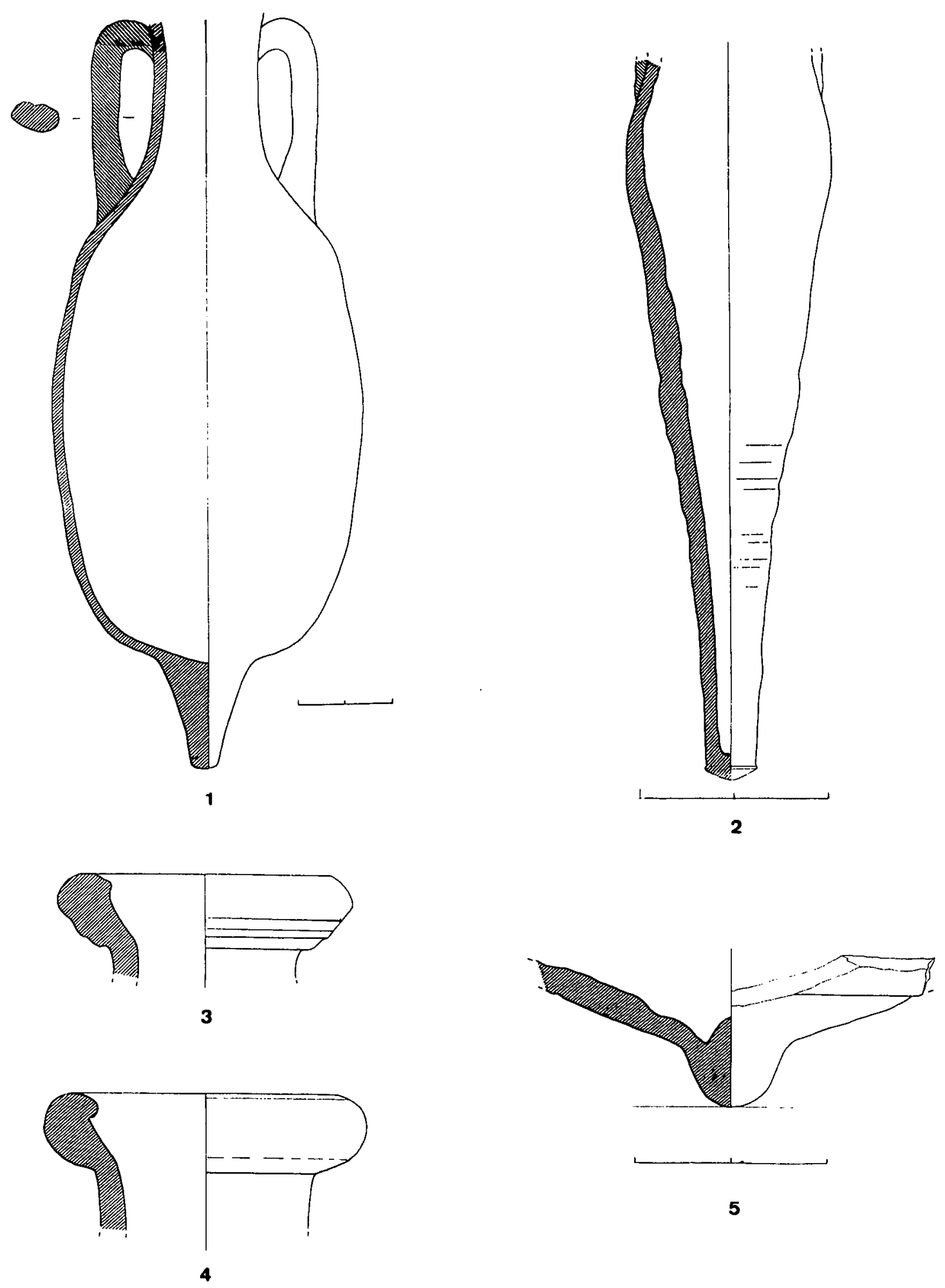

Fig. 2. Puente Melchor. Fase II: 1-5. 

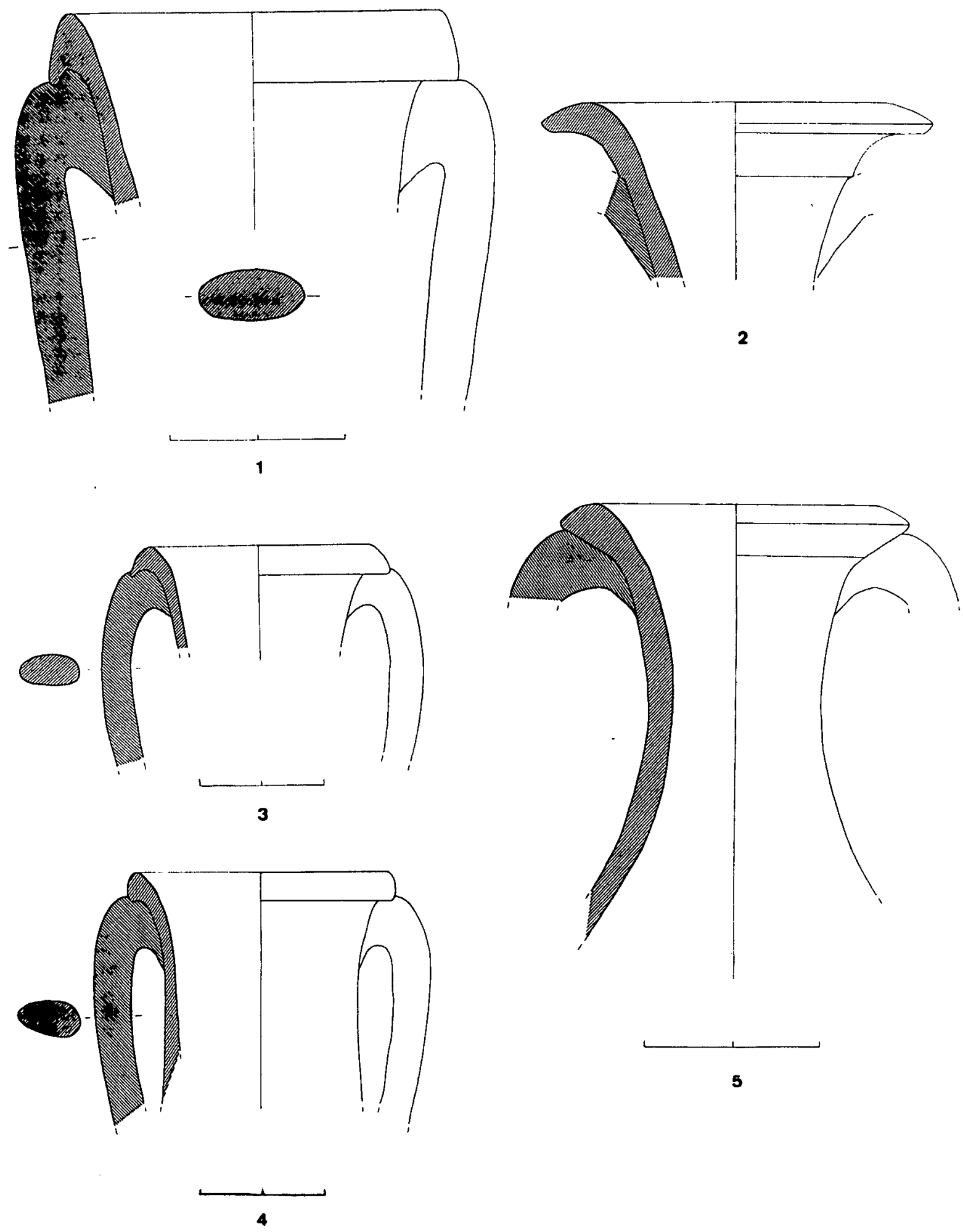

Fig. 3. Puente Melchor. Fases II: 1-2 y III: 3-5. 


$$
D_{-\infty}
$$




$$
\begin{aligned}
& 19 \\
& \phi
\end{aligned}
$$




$$
\begin{aligned}
& \text { P. ; } \\
& \text { d }
\end{aligned}
$$

